\title{
Nephrologist Interventions to Avoid Kidney Replacement Therapy in Acute Kidney Injury
}

\author{
Jonathan S. Chávez-Íñiguez ${ }^{a, b}$ Pablo Maggiani-Aguileraa, b \\ Christian Pérez-Flores ${ }^{a, b}$ Rolando Claure-Del Granado ${ }^{c, d}$ \\ Andrés E. De la Torre-Quiroga ${ }^{a, b}$ Alejandro Martínez-Gallardo González ${ }^{a, b}$ \\ Guillermo Navarro-Blackaller ${ }^{\mathrm{a}, \mathrm{b}}$ Ramón Medina-González ${ }^{\mathrm{a}}$ \\ Jochen G. Raimann ${ }^{e}$ Francisco G. Yanowsky-Escatell ${ }^{f}$ \\ Guillermo García-Garcíaa, b

\begin{abstract}
aNephrology Service, Civil Hospital of Guadalajara Fray Antonio Alcalde, Guadalajara, Mexico; ' University of Guadalajara Health Sciences Center, Guadalajara, Mexico; 'Division of Nephrology, Hospital Obrero \#2 - C.N.S., Cochabamba, Bolivia; dUniversidad Mayor de San Simon, School of Medicine, Cochabamba, Bolivia; eRenal Research Institute, New York, NY, USA; fDepartment of Internal Medicine, Hospital Civil de Guadalajara
\end{abstract} \\ Dr. Juan I. Menchaca, Guadalajara, Mexico
}

\section{Keywords}

Acute kidney injury · Hemodialysis · Mortality

\begin{abstract}
Background: Based on the pathophysiology of acute kidney injury (AKI), it is plausible that certain early interventions by the nephrologist could influence its trajectory. In this study, we investigated the impact of 5 early nephrology interventions on starting kidney replacement therapy (KRT), AKI progression, and death. Methods: In a prospective cohort at the Hospital Civil of Guadalajara, we followed up for 10 days AKI patients in whom a nephrology consultation was requested. We analyzed 5 early interventions of the nephrology team (fluid adjustment, nephrotoxic withdrawal, antibiotic dose adjustment, nutritional adjustment, and removal of hyperchloremic solutions) after the propensity score and multivariate analysis for the risk of starting KRT (primary objec-
\end{abstract}

tive), AKI progression to stage 3, and death (secondary objectives). Results: From 2017 to 2020, we analyzed 288 AKI patients. The mean age was 55.3 years, $60.7 \%$ were male, AKI KDIGO stage 3 was present in $50.5 \%$ of them, sepsis was the main etiology $50.3 \%$, and $72(25 \%)$ patients started KRT. The overall survival was $84.4 \%$. Fluid adjustment was the only intervention associated with a decreased risk for starting KRT (odds ratio [OR]: 0.58, 95\% confidence interval [Cl]: $0.48-$ 0.70 , and $p \leq 0.001$ ) and AKI progression to stage 3 (OR: 0.59, 95\% Cl: 0.49-0.71, and $p \leq 0.001)$. Receiving vasopressors and KRT were associated with mortality. None of the interventions studied was associated with reducing the risk of death. Conclusions: In this prospective cohort study of AKI patients, we found for the first time that early nephrologist intervention and fluid prescription adjustment were associated with lower risk of starting KRT and progression to AKI stage 3 . (c) 2021 The Author(s)

Published by S. Karger AG, Basel karger@karger.com www.karger.com/kbr

Karger $\stackrel{\text { ' }}{5}$

BOPEN ACCESS
(C) 2021 The Author(s)

Published by S. Karger AG, Basel

This is an Open Access article licensed under the Creative Commons Attribution-NonCommercial-4.0 International License (CC BY-NC) (http://www.karger.com/Services/OpenAccessLicense), applicable to the online version of the article only. Usage and distribution for commercial purposes requires written permission.
Correspondence to:

Jonathan S. Chávez-Íñiguez, jonarchi_10@ hotmail.com 


\section{Introduction}

The pathophysiology of acute kidney injury (AKI) is complex and associated with substantial morbidity and mortality $[1,2]$. AKI remains one of the largest risk factors for the development of chronic kidney disease (CKD) with possible progression into kidney replacement therapy (KRT) dependence and also mortality in patients admitted to intensive care units (ICUs) [3]. Despite great advances in the understanding of risk factors, diagnosis, and management of AKI, the risk of mortality remains high $[4,5]$. Early diagnosis and referral to specialized care by nephrologists have been shown to result in earlier adequate treatment of AKI. Delayed consultation of nephrologists associates with higher mortality [6-10], with statistics that reinforce the unarguable importance of early intervention. These interventions include optimization of fluid management, antibiotic dose and nutritional adjustments, nephrotoxic withdrawal, removal of hyperchloremic solutions, and others [11], all of which attempt to treat or ameliorate the various complications of AKI. Each of these has the potential to affect the course of AKI and the resulting outcomes. Despite the above being wellestablished facts, there is still a remarkable lack of data on the efficacy of each of the possible interventions. We aimed to identify the potential of each respective nephrology intervention to reduce the necessity to start KRT, the progression of $\mathrm{AKI}$, and mortality.

\section{Materials and Methods}

\section{Study Design and Patient Population}

A prospective, observational cohort study was conducted at the Hospital Civil de Guadalajara Fray Antonio Alcalde, Mexico, between August 2017 and March 2020. The hospital is a tertiary referral academic center with 1,709 beds. Studied patients were in the attention of the nephrology staff by consult request of the primary medical or surgical team. Only hospitalized patients in the ICU and wards with suspected AKI and under consult by the nephrology staff by request of the attending physician were included; due to the limited number of beds in the ICU, many patients who deserve to be in the ICU are managed in wards.

The initial request and the following treatment were fully at the discretion of the medical team and influenced by the study team. The responsible attending nephrologist ran daily rounds and was available $24 \mathrm{~h} /$ day. AKI was diagnosed based on the serum creatinine KDIGO criteria [11]. The inclusion criterion was early nephrology consultation, defined as consultation that occurred before $48 \mathrm{~h}$ after the AKI diagnosis. The exclusion criteria were CKD grade 5 , defined as a calculated glomerular filtration rate $<15 \mathrm{~mL} /$ $\mathrm{min} / 1.73 \mathrm{~m}^{2}$ by the 4 -variable Modification of Diet in Renal Disease Study-4 (MDRD-4) equation, chronic dialysis, hospitalization stay shorter than $48 \mathrm{~h}$, transplant patients, AKI diagnosis over 24 $\mathrm{h}$ after nephrology consultation, and missing data (unable to complete the analysis). A 10-day intramural follow-up period after AKI diagnosis was chosen retrospectively because all patients that started KRT did so within the first 10 days of follow-up [12]. The study was approved by the Hospital Civil de Guadalajara Fray Antonio Alcalde Institutional Review Board (HCG 146/18) and was conducted in adherence with the Declaration of Helsinki. Informed consent was obtained from all the subjects. The protocol followed the Strengthening the Reporting of Observational Studies in Epidemiology guidelines [13].

\section{Data Collection}

Demographic and clinical variables were collected as age, diabetes, hypertension, hypothyroidism, CKD stage (CKD was diagnosed as per the estimated glomerular filtration rate [eGFR] of $<60$ $\mathrm{mL} / \mathrm{min} / 1.73 \mathrm{~m}^{2}$, using the MDRD-4 equation) [11], smoking, cerebrovascular disease, and ischemic heart disease; cardiovascular, surgical, or medical hospital admission; drugs such as analgesics, antibiotics, antihypertensive medication, diuretics, vasopressors, statins, and aspirin during the course of hospitalization; the baseline serum creatinine level was defined as the most frequent value within a year before admission; contributing factors of AKI such as sepsis (Sepsis-3 criteria) [14], clinically assessed hypovolemia, cardiorenal syndrome [15], nephrotoxic drugs and shock; prespecified biochemical data such as hemoglobin, platelets, leukocytes, glucose, urea, creatinine, sodium, potassium, chloride, phosphate, calcium, arterial $\mathrm{pH}, \mathrm{PCO}_{2}, \mathrm{PO}_{2}$, bicarbonate, and lactate levels.

\section{Nephrology Interventions}

Early nephrology interventions were performed following the KDIGO guidelines [11] and expert panel recommendations [10]; we focused on 5 prespecified interventions as follows: (1) fluid adjustment, (2) nephrotoxic withdrawal, (3) antibiotic dose adjustment according to eGFR by MDRD-4, (4) nutritional adjustment, and (5) removal of hyperchloremic solutions. Fluid adjustment was defined as the use of fluids according to the clinical scenario and/or with point of care ultrasound evaluation of the inferior caval vein (diameter and collapsibility index or the pulse pressure variability) and lungs for the presence of pleural B lines, in the cases where the device was available. In those with suspected clinical hypovolemia, isotonic crystalloids were used; in those where there was no need for intravenous fluids or there was a risk of fluid overload, fluids, starches, and dextrans were reduced or suspended, respectively. Following or concomitant with fluid resuscitation, hypotensive patients were given vasoconstrictor, most commonly norepinephrine, titrated to a target mean arterial pressure greater than $65 \mathrm{~mm} \mathrm{Hg}$. Nephrotoxic withdrawal was defined as the cessation of those drugs known to be nephrotoxic according to KDIGO AKI guidelines and expert panel recommendations $[11,16,17]$. Antibiotic dose adjustment was defined as the dose adjustment by the eGFR according to the MDRD-4 equation $[14,18]$. Nutritional adjustment was defined as nutritional adjustment according to the KDIGO AKI guidelines, and the recommendation was $25-30 \mathrm{kcal} / \mathrm{kg} /$ day with protein requirements of at least 1.0 up to $1.7 \mathrm{~g} / \mathrm{kg} /$ day [11]. Removal of hyperchloremic solutions was defined as the removal or replacement of hyperchloremic fluid, such as changing $0.9 \%$ saline to Ringer lactate or glucose $5 \%$ (in patients with hypernatremia or serum sodium $>145 \mathrm{mmol} / \mathrm{L}$ ). 


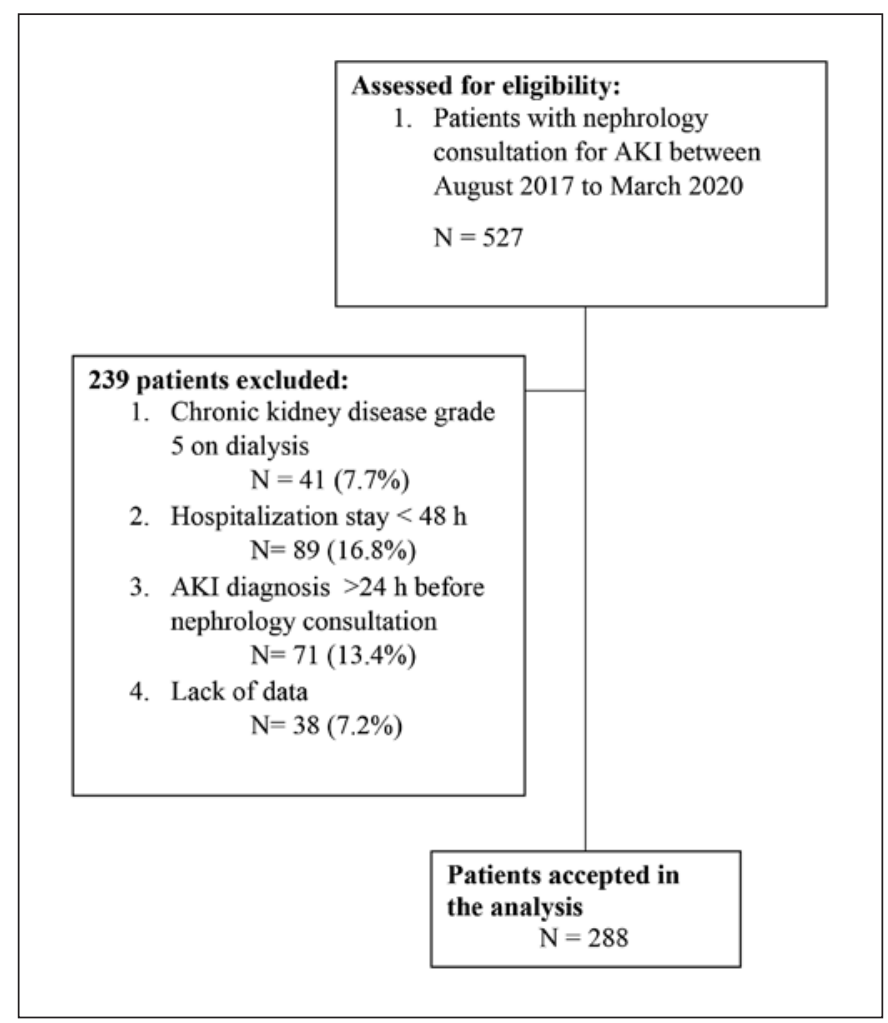

Fig. 1. Flowchart of study population. AKI, acute kidney injury; $\mathrm{CKD}$, chronic kidney disease.

Classic indications for KRT were fluid overload-resistant to diuretics, severe hyperkalemia, severe metabolic acidosis, and uremic manifestations, including encephalopathy, pericarditis, and convulsion $[11,19,20]$. Data were collected by 2 observers who were not involved in patient care.

\section{Study Outcomes}

The primary outcome was to determine which of the predefined early nephrology interventions reduces the necessity to start KRT. The secondary outcomes were to determine whether variables were associated with AKI progression (defined as worsening stage of AKI) and mortality, as well as if there was an association between early nephrology intervention and these outcomes. As an exploratory analysis, we wanted to identify which of the classic KRT indications in AKI patients (fluid overload, hyperkalemia, acid-base disorders, and uremia) were independently associated with starting KRT and mortality.

\section{Statistical Analysis}

Categorical descriptive data are presented as frequencies and continuous variables as the means \pm SDs and percentages (\%). The Shapiro-Wilk test was used to determine the distribution of the variables that presented an abnormal distribution, so it was decided to use the Wilcoxon signed-rank test to determine the differences between the variables. Survival probabilities for the tested groups were assessed using the construction of Kaplan-Meier survival curves and comparison by the log-rank test. To assemble comparable groups, we employed the nearest neighbor propensity score matching in a 1:1 fashion for the comparison was applied to compare AKI outcomes with early nephrology interventions. A univariate and multivariate binary logistic regression model was used to determine the variables associated with KRT, AKI progression, and mortality during the first 10-day follow-up, adjusted for age, sex, diabetes, hypertension, smoking, hypothyroidism, CKD, cerebrovascular and ischemic heart diseases, analgesics, antibiotics, antihypertensives, diuretics, vasopressors, body mass index, sepsis, hypovolemia, cardiorenal syndrome, nephrotoxic drugs, shock, liquid, antibiotic dose and nutritional adjustment, nephrotoxic withdrawal, removal of hyperchloremic solutions, and AKI grade. The results are reported as odds ratios (ORs) and the corresponding confidence intervals (CIs). ORs that do not cross unit and $p<0.05$ were considered statistically significant. R Studio pro$\operatorname{gram}^{\circledR}$ (version 1.1.38, 2017) was used for data analyses.

\section{Results}

\section{Baseline Characteristics Associated with KRT}

Between August 2017 and March 2020, a total of 527 patients were referred to nephrology consultation for suspected AKI during their hospitalization. We excluded 41 (7.7\%) patients with CKD grade 5 on dialysis, $89(16.8 \%)$ with a hospitalization stay shorter than $48 \mathrm{~h}, 71$ (13.4\%) with AKI diagnosis more than $24 \mathrm{~h}$ before nephrology consultation, and 38 (7.2\%) patients with lack of data, resulting in 288 patients included in the final analysis as shown in Figure 1. Baseline characteristics of patients according to KRT groups are shown in Table 1. Sixty (60\%) were males, with a mean age of $55.3 \pm 18.3$ years. A total of $116(40.2 \%)$ patients had diabetes; 16 (5.5\%) had hypothyroidism; 147 (51.2\%) had a medical noncardiovascular or surgical hospital admission cause; 165 (57.2\%) were receiving analgesics, $221(76.7 \%)$ antibiotics, and 117 (40.6\%) received diuretics during hospitalization. AKI KDIGO 3 was present in $145(50.5 \%)$ patients, and the main contributors to AKI were sepsis (50.3\%) and hypovolemia (45.8\%). Fluid adjustment was the principal nephrologist nondialytic intervention in $238(82.6 \%)$ patients. A total of $72(25 \%)$ patients needed KRT, and 45 (15.6\%) patients died during the follow-up. AKI patients requiring KRT presented more often with hypothyroidism, presented less frequently with cardiovascular and surgical hospital admission, had an increased use of vasopressors, had more severe AKI KDIGO grades, and had fluid adjustment and nephrotoxic withdrawal less often and had a higher mortality than patients who did not need KRT as shown in Table 1. The overall survival for the 10 -day follow-up was $84.4 \%$ (95\% CI: 0.80 0.88 ) is shown in online suppl. Figure 1; for all online suppl. material, see www.karger.com/doi/10.1159/000517615. 
Table 1. Baseline demographic, clinical, and laboratory characteristics of patients according to KRT groups

\begin{tabular}{|c|c|c|c|c|}
\hline Variable & Total & KRT & Non-KRT & $p$ value \\
\hline$N(\%)$ & 288 & $72(25)$ & $216(75)$ & \\
\hline Age, years, mean (SD) & $55.3(18.3)$ & $51.8(18.3)$ & $56.4(18.1)$ & 0.07 \\
\hline \multicolumn{5}{|l|}{ Gender, $N(\%)$} \\
\hline Male & $175(60.7)$ & $40(55.5)$ & $135(62.5)$ & 0.29 \\
\hline Female & $113(39.3)$ & $32(44.5)$ & $81(37.5)$ & \\
\hline BMI, kg/m², mean (SD) & $26.3(6.3)$ & $26.7(7.0)$ & $26.1(6.1)$ & 0.49 \\
\hline \multicolumn{5}{|l|}{ Comorbidities, $N(\%)$} \\
\hline Diabetes & $116(40.2)$ & $27(37.5)$ & $89(41.2)$ & 0.58 \\
\hline Hypertension & $126(43.7)$ & $29(40.2)$ & $97(44.9)$ & 0.49 \\
\hline Smoker & $55(19.0)$ & $11(15.2)$ & $44(20.3)$ & 0.34 \\
\hline Hypothyroidism & $16(5.5)$ & $8(11.1)$ & $8(3.7)$ & 0.01 \\
\hline CKD grade $1-4$ & $98(34.0)$ & $22(30.5)$ & $76(35.1)$ & 0.47 \\
\hline Cerebrovascular disease & $12(4.1)$ & $3(4.1)$ & $9(4.1)$ & 1.00 \\
\hline Ischemic heart disease & $10(3.4)$ & $1(1.3)$ & $9(4.1)$ & 0.26 \\
\hline \multicolumn{5}{|l|}{ Hospital admission, $N(\%)$} \\
\hline Cardiovascular & $46(15.9)$ & $10(13.8)$ & $36(16.6)$ & 0.57 \\
\hline Surgical & $95(32.9)$ & $17(23.6)$ & $78(36.1)$ & 0.06 \\
\hline Other medical & $147(51.2)$ & $45(62.6)$ & $102(47.3)$ & 0.02 \\
\hline \multicolumn{5}{|l|}{ Hospitalization drugs, $N(\%)$} \\
\hline Analgesics & $165(57.2)$ & $44(61.1)$ & $121(56.0)$ & 0.45 \\
\hline Antibiotics & $221(76.7)$ & $58(80.5)$ & $163(75.4)$ & 0.37 \\
\hline Antihypertensive & $82(28.4)$ & $19(26.3)$ & $63(29.1)$ & 0.65 \\
\hline Diuretics & $117(40.6)$ & $34(47.2)$ & $83(38.4)$ & 0.18 \\
\hline Vasopressors & $79(27.4)$ & $29(40.2)$ & $50(23.1)$ & 0.004 \\
\hline Statins & $49(17.0)$ & $9(12.5)$ & $40(18.5)$ & 0.24 \\
\hline Aspirin & $49(17.0)$ & $9(12.5)$ & $40(18.5)$ & 0.24 \\
\hline \multicolumn{5}{|l|}{ AKI grade, $N(\%)$} \\
\hline KDIGO-1 & $65(22.5)$ & $6(8.3)$ & $59(27.3)$ & $<0.001$ \\
\hline KDIGO-2 & $78(27.0)$ & $8(11.1)$ & $70(32.4)$ & $<0.001$ \\
\hline KDIGO-3 & $145(50.5)$ & $58(80.6)$ & $87(40.3)$ & $<0.001$ \\
\hline \multicolumn{5}{|c|}{ Contributing factors to AKI, $N(\%)$} \\
\hline Sepsis & $145(50.3)$ & $44(61.1)$ & $101(46.7)$ & 0.03 \\
\hline Hypovolemia & $132(45.8)$ & $28(38.8)$ & $104(48.1)$ & 0.17 \\
\hline Cardiorenal syndrome & $34(11.8)$ & $5(6.9)$ & $29(13.4)$ & 0.14 \\
\hline Nephrotoxic drugs & $52(18.0)$ & $13(18.0)$ & $39(18.0)$ & 1.00 \\
\hline Shock & $92(31.9)$ & $31(43.0)$ & $61(28.2)$ & 0.01 \\
\hline \multicolumn{5}{|l|}{ Biochemical data, mean (SD) } \\
\hline Serum hemoglobin, g/dL & $10.2(2.3)$ & $9.9(1.9)$ & $10.3(2.5)$ & 0.33 \\
\hline Serum platelets, $10^{9} / \mathrm{L}$ & $207.5(134.8)$ & $175.3(92.2)$ & $218.2(155.1)$ & 0.02 \\
\hline Serum leukocytes, $10^{9} / \mathrm{L}$ & $12.6(6.7)$ & $14.4(7.5)$ & $12.0(7.2)$ & 0.03 \\
\hline Serum glucose, mg/dL & $129.6(56.6)$ & $122.3(58.5)$ & $132.0(76.5)$ & 0.19 \\
\hline Serum urea, $\mathrm{mg} / \mathrm{dL}$ & $151.6(80.9)$ & $184.5(91.1)$ & $140.7(87.3)$ & $<0.001$ \\
\hline $\mathrm{SCr}, \mathrm{mg} / \mathrm{dL}$ & $3.9(2.8)$ & $5.4(2.9)$ & $3.4(2.5)$ & $<0.001$ \\
\hline Serum sodium, mEq/L & $136.6(7.1)$ & $135.3(8.8)$ & $137.1(9.7)$ & 0.07 \\
\hline Serum potassium, $\mathrm{mEq} / \mathrm{L}$ & $4.5(0.8)$ & $4.8(2.1)$ & $4.4(2.0)$ & 0.001 \\
\hline Serum chloride, $\mathrm{mEq} / \mathrm{L}$ & $102.7(13.5)$ & $99.5(12.1)$ & $103.8(14.3)$ & 0.09 \\
\hline Serum phosphate, mg/dL & $5.4(2.3)$ & $6.8(3.2)$ & $4.9(2.7)$ & 0.51 \\
\hline Serum calcium, mg/dL & $8.0(6.1)$ & $9.0(7.1)$ & $7.7(3.5)$ & 0.93 \\
\hline Arterial $\mathrm{pH}$ & $7.34(0.09)$ & $7.33(0.06)$ & $7.35(0.10)$ & 0.12 \\
\hline $\mathrm{PCO}_{2}, \mathrm{~mm} \mathrm{Hg}$ & $34.5(16.5)$ & $35.7(14.4)$ & $34.1(18.1)$ & 0.98 \\
\hline $\mathrm{PO}_{2}, \mathrm{~mm} \mathrm{Hg}$ & $72.0(42.6)$ & $79.7(33.8)$ & $69.6(43.2)$ & 0.55 \\
\hline Serum bicarbonate, $\mathrm{mEq} / \mathrm{L}$ & $19.4(9.4)$ & $18.5(7.4)$ & $19.3(10.5)$ & 0.20 \\
\hline Serum lactate, $\mathrm{mmol} / \mathrm{L}$ & $3.1(3.2)$ & $2.5(1.3)$ & $3.3(2.1)$ & 0.54 \\
\hline
\end{tabular}


Table 1 (continued)

\begin{tabular}{|c|c|c|c|c|}
\hline Variable & Total & KRT & Non-KRT & $p$ value \\
\hline \multicolumn{5}{|c|}{ Nephrologist nondialysis intervention, $N(\%)$} \\
\hline Fluid adjustment & $238(82.6)$ & $33(45.8)$ & $205(94.9)$ & $<0.001$ \\
\hline Nephrotoxic withdrawal & $73(25.3)$ & $8(11.1)$ & $65(30.0)$ & 0.001 \\
\hline Antibiotic dose adjustment & $46(15.9)$ & $9(12.5)$ & $37(17.1)$ & 0.35 \\
\hline Nutritional adjustment & $8(2.7)$ & $0(0.0)$ & $8(3.7)$ & 0.09 \\
\hline Remove hyperchloremic solutions & $13(4.5)$ & $1(1.3)$ & $12(5.5)$ & 0.14 \\
\hline \multicolumn{5}{|l|}{$\mathrm{KRT}, N(\%)$} \\
\hline Hemodialysis & $66(22.9)$ & $66(91.6)$ & - & - \\
\hline Peritoneal dialysis & $6(2.1)$ & $6(8.4)$ & - & - \\
\hline \multicolumn{5}{|l|}{ Cause of KRT, $N(\%)$} \\
\hline Hyperkalemia & $21(7.2)$ & $21(29.1)$ & - & - \\
\hline Acid-base disorders & $24(8.3)$ & $24(33.3)$ & - & - \\
\hline Fluid overload & $44(15.2)$ & $44(61.1)$ & - & - \\
\hline Uremia & $26(9.0)$ & $26(36.1)$ & - & - \\
\hline Mortality, N (\%) & $45(15.6)$ & $20(27.7)$ & $25(11.5)$ & 0.001 \\
\hline
\end{tabular}

AKI, acute kidney injury; CKD, chronic kidney disease; KDIGO; Kidney Disease Initiative Global Outcomes; KRT, kidney replacement therapy; BMI, body mass index; SCr, serum creatinine.

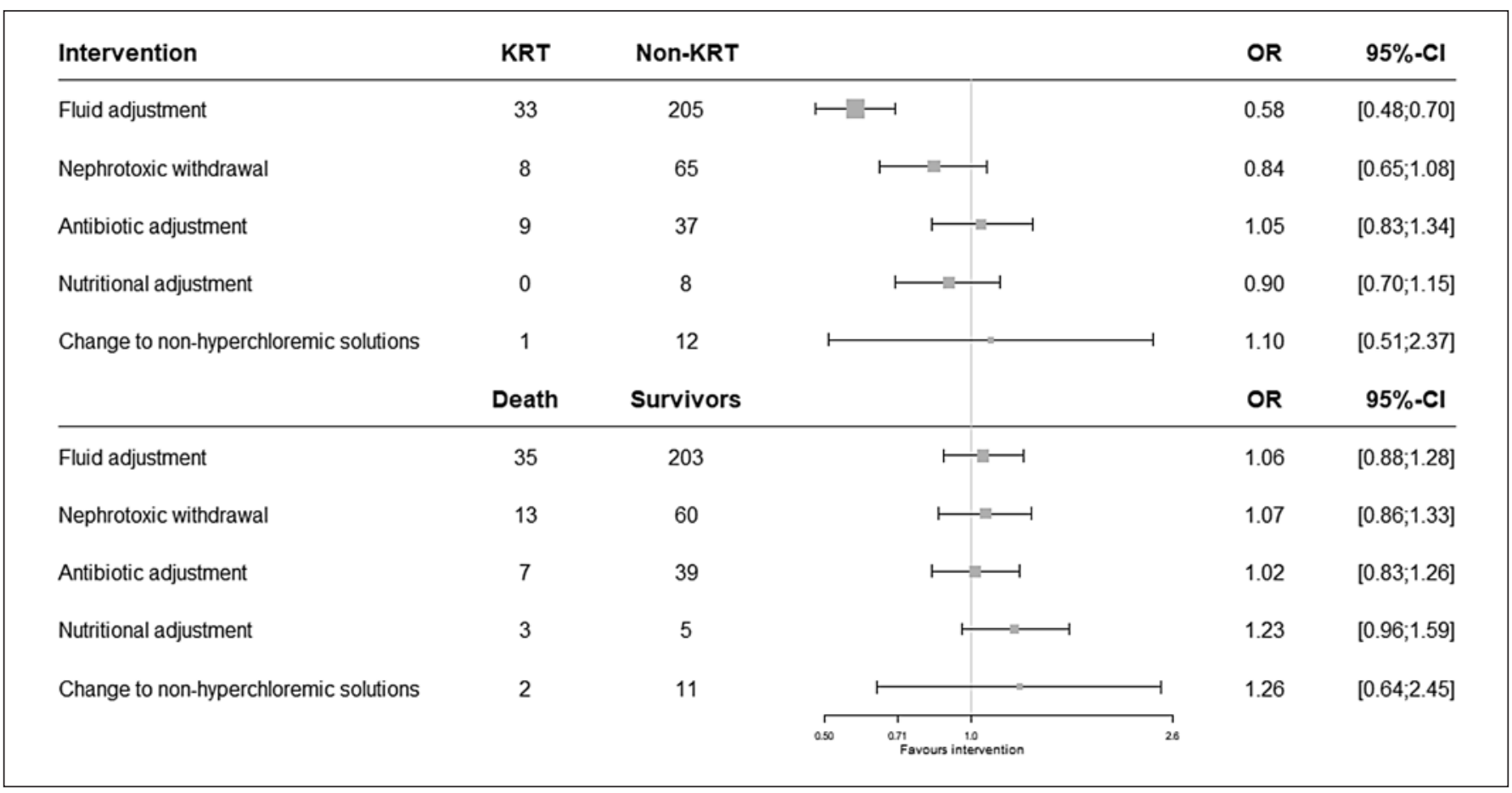

Fig. 2. Forest plot of early nephrologist intervention associated with KRT and death. KRT, kidney replacement therapy; OR, odds ratio; CI, confidence interval.

Primary Outcome, Nephrologist Interventions, and Their Impact on the Initiation of KRT

A multivariate analysis showed that variables associated with an increased risk for KRT were hypothyroidism
(OR: $1.19,95 \%$ CI: $1.00-1.42$, and $p=0.04$ ), diuretics (OR: $1.18,95 \%$ CI: $1.08-1.30$, and $p=0.001$ ), and AKI KDIGO 3 (OR: 1.25, 95\% CI: $1.13-1.37$, and $p \leq 0.001$ ). In an attempt to identify variables and nephrologist inter- 
Table 2. Univariable-multivariable logistic regression model to determine the variables associated with start KRT in AKI patients before propensity score analysis

\begin{tabular}{|c|c|c|c|c|}
\hline & Univariate $(95 \% \mathrm{CI})$ & $p$ value & Multivariate (95\% CI) & $p$ value \\
\hline Age & $0.99(0.99-1.00)$ & 0.64 & $0.99(0.99-1.00)$ & 0.06 \\
\hline Female & $0.98(0.83-1.16)$ & 0.86 & $1.09(0.88-1.33)$ & 0.40 \\
\hline Type 2 diabetes mellitus & $0.97(0.87-1.07)$ & 0.58 & $1.07(0.97-1.19)$ & 0.14 \\
\hline Hypertension & $1.01(0.85-1.20)$ & 0.86 & $1.02(0.82-1.26)$ & 0.85 \\
\hline Smoker & $0.95(0.76-1.18)$ & 0.65 & $0.99(0.79-1.24)$ & 0.95 \\
\hline Hypothyroidism & $1.03(0.79-1.35)$ & 0.78 & $1.11(0.85-1.46)$ & 0.43 \\
\hline CKD grade $1-4$ & $1.01(0.84-1.21)$ & 0.85 & $0.91(0.75-1.11)$ & 0.37 \\
\hline Cerebrovascular disease & $1.10(0.70-1.73)$ & 0.65 & $1.25(0.78-2.02)$ & 0.34 \\
\hline Ischemic heart disease & $0.84(0.47-1.49)$ & 0.56 & $0.75(0.40-1.41)$ & 0.38 \\
\hline BMI & $0.99(0.98-1.01)$ & 0.90 & $0.99(0.98-1.01)$ & 0.59 \\
\hline Sepsis & $1.10(0.93-1.30)$ & 0.24 & $1.03(0.86-1.24)$ & 0.69 \\
\hline Hypovolemia & $1.01(0.85-1.20)$ & 0.86 & $0.97(0.80-1.17)$ & 0.77 \\
\hline Cardiorenal syndrome & $0.77(0.60-0.99)$ & 0.04 & $0.83(0.61-1.13)$ & 0.24 \\
\hline Nephrotoxic drugs & $1.00(0.80-1.23)$ & 1.00 & $1.02(0.81-1.29)$ & 0.81 \\
\hline Shock & $1.13(1.02-1.26)$ & 0.01 & $1.03(0.92-1.15)$ & 0.58 \\
\hline Vasoactive drugs & $1.07(0.90-1.27)$ & 0.39 & $1.14(0.94-1.38)$ & 0.16 \\
\hline AKI KDIGO 1 & $0.76(0.61-0.95)$ & 0.02 & $1.01(0.74-1.37)$ & 0.94 \\
\hline AKI KDIGO 2 & $0.96(0.74-1.24)$ & 0.79 & 1.00 & 1.00 \\
\hline AKI KDIGO 3 & $1.21(1.01-1.45)$ & 0.03 & $1.28(0.99-1.66)$ & 0.053 \\
\hline NSAIDs & $1.04(0.88-1.23)$ & 0.61 & $1.06(0.88-1.27)$ & 0.51 \\
\hline Antibiotics & $1.06(0.87-1.29)$ & 0.54 & $1.01(0.80-1.27)$ & 0.90 \\
\hline Antihypertensive & $0.98(0.81-1.18)$ & 0.85 & $1.04(0.85-1.28)$ & 0.67 \\
\hline Diuretics & $1.07(0.79-0.96)$ & 0.01 & $1.23(1.00-1.50)$ & 0.04 \\
\hline Fluid adjustment & $0.61(0.52-0.71)$ & $<0.001$ & $0.58(0.48-0.70)$ & $<0.001$ \\
\hline Nephrotoxic withdrawal & $0.83(0.66-1.04)$ & 0.11 & $0.84(0.65-1.08)$ & 0.19 \\
\hline Antibiotic adjustment & $0.89(0.71-1.12)$ & 0.35 & $1.05(0.83-1.34)$ & 0.64 \\
\hline Nutritional adjustment & $0.77(0.57-1.04)$ & 0.09 & $0.90(0.70-1.15)$ & 0.41 \\
\hline Remove hyperchloremic solutions & $1.00(0.49-2.01)$ & 1.00 & $1.10(0.51-2.37)$ & 0.80 \\
\hline
\end{tabular}

AKI, acute kidney injury; KDIGO; Kidney Disease Initiative Global Outcomes; NSAIDs, nonsteroidal analgesic anti-inflammatory drugs; CKD, chronic kidney disease; BMI, body mass index; CI, confidence interval; KRT, kidney replacement therapy.

ventions associated with the initiation of KRT in AKI patients at the 10-day follow-up, a decreased risk was fluid adjustment (OR: 0.57, 95\% CI: $0.51-0.64$, and $p \leq 0.001$ ) and nephrotoxic withdrawal (OR: $0.87,95 \%$ CI: $0.79-$ 0.96 , and $p=0.01$ ) as shown in online suppl. Table 1 .

\section{Propensity Score-Matching Analysis}

A propensity score-matching analysis on sex and age was further applied to analyze the potential impact of nephrologist interventions on outcomes in AKI patients; it would be enough to match the groups sufficiently well based on the existing evidence that the 2 variables carry different risks for the development and evolution of AKI. After this analysis, 72 patients with fluid adjustment were compared with 72 patients treated with other interventions. The main prognostic covariables were properly bal- anced between subgroups after propensity score-matching analysis, and no significant differences were observed (online suppl. Fig. 2, 3). Consistent with the results for the entire cohort, diuretics increased the risk of KRT (OR: 1.23 , 95\% CI: $1.00-1.50$, and $p<0.004$ ), and fluid adjustment decreased the risk (OR: $0.58,95 \%$ CI: $0.48-0.70$, and $p<0.001$ ), even after adjusting for potential confounders as shown in Table 2 and Figure 2. Similar results were observed with secondary outcomes.

\section{Secondary Outcomes, Variables Associated with AKI}

Progression, and Mortality according to Nephrologist Intervention

Nephrologist fluid adjustment (OR: 0.59, 95\% CI: $0.49-0.71$, and $p \leq 0.001$ ) was the only variable associated with a reduction in AKI progression to KDIGO stage 3 
Table 3. Univariable-Multivariable logistic regression model to determine the variables associated with AKI progression to stage 3 at 10-day follow-up before propensity score analysis

\begin{tabular}{lcccc}
\hline & Univariate $(95 \% \mathrm{CI})$ & $p$ value & Multivariate (95\% CI) $p$ value \\
\hline Age & $0.99(0.99-1.00)$ & 0.64 & $0.99(0.99-1.00)$ & 0.14 \\
Female & $0.94(0.83-1.06)$ & 0.34 & $0.90(0.79-1.02)$ & 0.10 \\
Type 2 diabetes mellitus & $0.98(0.83-1.16)$ & 0.86 & $1.05(0.85-1.29)$ & 0.61 \\
Hypertension & $1.01(0.85-1.20)$ & 0.86 & $1.06(0.86-1.32)$ & 0.54 \\
Smoker & $0.95(0.76-1.18)$ & 0.65 & $1.00(0.79-1.25)$ & 0.99 \\
Hypothyroidism & $1.03(0.79-1.35)$ & 0.78 & $1.03(0.79-1.36)$ & 0.78 \\
CKD grade 1-4 & $1.01(0.84-1.21)$ & 0.85 & $0.93(0.76-1.13)$ & 0.51 \\
Cerebrovascular disease & $1.10(0.70-1.73)$ & 0.65 & $1.38(0.85-2.24)$ & 0.18 \\
Ischemic heart disease & $0.84(0.47-1.49)$ & 0.56 & $0.76(0.40-1.45)$ & 0.41 \\
BMI & $0.99(0.98-1.01)$ & 0.90 & $0.99(0.98-1.01)$ & 0.56 \\
Sepsis & $1.10(0.93-1.30)$ & 0.24 & $1.04(0.86-1.26)$ & 0.64 \\
Hypovolemia & $1.01(0.85-1.20)$ & 0.86 & $0.99(0.81-1.20)$ & 0.93 \\
Cardiorenal syndrome & $0.77(0.60-0.99)$ & 0.04 & $0.82(0.60-1.12)$ & 0.21 \\
Nephrotoxic drugs & $1.00(0.80-1.23)$ & 1.00 & $1.04(0.83-1.32)$ & 0.52 \\
Shock & $0.96(0.85-1.09)$ & 0.55 & $0.86(0.72-1.02)$ & 0.09 \\
Vasoactive drugs & $1.07(0.90-1.27)$ & 0.39 & $1.11(0.91-1.34)$ & 0.27 \\
NSAIDs & $1.04(0.88-1.23)$ & 0.61 & $1.05(0.87-1.27)$ & 0.56 \\
Antibiotics & $1.06(0.87-1.29)$ & 0.54 & $1.02(0.81-1.29)$ & 0.82 \\
Antihypertensive & $0.98(0.81-1.18)$ & 0.85 & $1.03(0.84-1.28)$ & 0.71 \\
Diuretics & $0.95(0.81-1.13)$ & 0.62 & $1.15(0.94-1.41)$ & 0.15 \\
Fluid adjustment & $0.61(0.52-0.71)$ & $<0.001$ & $0.59(0.49-0.71)$ & $<0.001$ \\
Nephrotoxic withdrawal & $0.83(0.66-1.04)$ & 0.11 & $0.88(0.68-1.13)$ & 0.33 \\
Antibiotic adjustment & $0.89(0.71-1.12)$ & 0.35 & $1.04(0.81-1.32)$ & 0.74 \\
Nutritional adjustment & $0.77(0.54-1.09)$ & 0.14 & $0.75(0.52-1.07)$ & 0.12 \\
Remove hyperchloremic solutions & $1.00(0.49-2.01)$ & 1.00 & $1.28(0.59-2.78)$ & 0.52 \\
\hline & & & & \\
\hline
\end{tabular}

NSAIDs, nonsteroidal analgesic anti-inflammatory drugs; AKI, acute kidney injury; CKD, chronic kidney disease; BMI, body mass index; CI, confidence interval.

after the score matching and multivariable analysis is shown in Table 3. Vasopressors (OR: 1.25, 95\% CI: 1.061.47 , and $p=0.008$ ) and the need for KRT (OR: 1.15, 95\% CI: $1.01-1.35$, and $p=0.04$ ) increased the mortality risk at the 10-day follow-up as shown in Table 4, after the score matching and multivariable analysis.

\section{Exploratory Analysis, Identification of Variables}

Associated with the Classic Indication to Initiate KRT, and Mortality

Among the classic indications to initiate KRT in AKI patients as having fluid overload, hyperkalemia, acidbase disorders, and uremia, we found that fluid overload was the main cause that led to starting KRT $(1.91,95 \% \mathrm{CI}$ : $1.75-2.08$, and $p<0.001)$, followed by uremia $(1.46,95 \%$ CI: $1.31-1.63$, and $p<0.001)$ in multivariable analysis as shown in online suppl. Table 2 . We also found that fluid overload was the only factor significantly associated with mortality $(1.13,95 \%$ CI: $1.00-1.27$, and $p=0.04)$.

Nephrologist Interventions to Avoid KRT in AKI

\section{Discussion}

In this single-center prospective cohort study in AKI patients, we showed that fluid adjustment was an early nephrologist intervention that could reduce the risk of starting KRT and reduce AKI progression to KDIGO grade 3 . We found that fluid adjustment was associated with a $42 \%$ reduction in the probability of starting KRT. Fluid adjustment may be one of the most relevant strategies in AKI patients [21, 22]. It is a spectrum of interventions that have different positive effects on the injured kidney. For example, in patients with hypovolemia, a frequent etiology of AKI [23], timely fluid administration may be a preventive measure against $\mathrm{AKI}$ and should be effective both through restoring the circulating volume, improving the impaired renal perfusion, and promoting the recovery of kidney functions [24]. In AKI, the rationale of fluid therapy is to restore the mean arterial pressure (which determines renal perfusion pressure) and 
Table 4. Univariable-Multivariable logistic regression model to determine the variables associated with mortality at 10-day follow-up in AKI patients before propensity score analysis

\begin{tabular}{|c|c|c|c|c|}
\hline & Univariate $(95 \% \mathrm{CI})$ & $p$ value & Multivariate $(95 \% \mathrm{CI})$ & $p$ value \\
\hline Age & $1.00(0.99-1.00)$ & 0.29 & $1.00(0.99-1.00)$ & 0.20 \\
\hline Female & $0.96(0.88-1.04)$ & 0.37 & $0.93(0.85-1.01)$ & 0.28 \\
\hline Type 2 diabetes mellitus & $0.91(0.80-1.05)$ & 0.23 & $0.94(0.79-1.12)$ & 0.53 \\
\hline Hypertension & $0.90(0.79-1.04)$ & 0.17 & $0.96(0.80-1.16)$ & 0.71 \\
\hline Smoker & $1.04(0.86-1.24)$ & 0.65 & $1.04(0.86-1.27)$ & 0.64 \\
\hline Hypothyroidism & $1.05(0.84-1.32)$ & 0.61 & $1.02(0.81-1.30)$ & 0.82 \\
\hline CKD grade $1-4$ & $0.92(0.80-1.07)$ & 0.32 & $0.99(0.83-1.17)$ & 0.94 \\
\hline Cerebrovascular disease & $0.98(0.68-1.42)$ & 0.93 & $1.14(0.75-1.72)$ & 0.53 \\
\hline Ischemic heart disease & $0.80(0.50-1.28)$ & 0.36 & $0.76(0.44-1.31)$ & 0.33 \\
\hline BMI & $0.99(0.98-1.00)$ & 0.41 & $0.99(0.98-1.01)$ & 0.77 \\
\hline Sepsis & $1.01(0.88-1.16)$ & 0.81 & $0.91(0.78-1.07)$ & 0.30 \\
\hline Hypovolemia & $0.94(0.82-1.08)$ & 0.44 & $0.98(0.83-1.16)$ & 0.87 \\
\hline Cardiorenal syndrome & $0.88(0.72-1.08)$ & 0.25 & $0.95(0.73-1.25)$ & 0.75 \\
\hline Nephrotoxic drugs & $1.17(0.98-1.39)$ & 0.07 & $1.09(0.89-1.33)$ & 0.37 \\
\hline Vasoactiver drugs & $1.37(1.20-1.56)$ & $<0.001$ & $1.25(1.06-1.47)$ & 0.008 \\
\hline AKI KDIGO 1 & $0.91(0.75-1.09)$ & 0.33 & $1.00(0.76-1.31)$ & 0.97 \\
\hline AKI KDIGO 2 & $1.02(0.82-1.26)$ & 0.83 & 1.00 & 1.00 \\
\hline AKI KDIGO 3 & $1.05(0.90-1.22)$ & 0.52 & $1.00(0.80-1.26)$ & 0.95 \\
\hline NSAIDs & $0.88(0.77-1.01)$ & 0.07 & $0.87(0.74-1.02)$ & 0.10 \\
\hline Antibiotics & $1.21(1.03-1.42)$ & 0.02 & $1.12(0.91-1.36)$ & 0.26 \\
\hline Antihypertensive & $0.85(0.73-0.99)$ & 0.04 & $0.92(0.77-1.10)$ & 0.40 \\
\hline Diuretics & $0.93(0.82-1.07)$ & 0.35 & $0.97(0.81-1.16)$ & 0.79 \\
\hline KRT & $1.13(1.00-1.29)$ & 0.05 & $1.15(1.01-1.35)$ & 0.04 \\
\hline Fluid adjustment & $1.00(0.86-1.15)$ & 0.95 & $1.06(0.88-1.28)$ & 0.49 \\
\hline Nephrotoxic withdrawal & $1.11(0.92-1.33)$ & 0.26 & $1.07(0.86-1.33)$ & 0.51 \\
\hline Antibiotic adjustment & $1.07(0.88-1.29)$ & 0.48 & $1.02(0.83-1.26)$ & 0.81 \\
\hline Nutritional adjustment & & & $1.23(0.96-1.59)$ & 0.10 \\
\hline Remove hyperchloremic solutions & $1.33(0.75-2.37)$ & 0.32 & $1.26(0.64-2.45)$ & 0.49 \\
\hline
\end{tabular}

AKI, acute kidney injury; CKD, chronic kidney disease; KDIGO, Kidney Disease Initiative Global Outcomes; NSAIDs, nonsteroidal analgesic anti-inflammatory drugs; BMI, body mass index; CI, confidence interval; KRT, kidney replacement therapy.

cardiovascular output (required for adequate renal blood flow). There is a fine line between achieving proper fluid resuscitation and fluid overload; unfortunately, the kidney is particularly affected by fluid overload, which can lead to increased renal subcapsular pressure and lowered renal blood flow and glomerular filtration rate [25]. Cautious fluid administration guided by markers of fluid responsiveness can be considered [26] because excessive fluid administration should be avoided to prevent harmful fluid overload $[27,28]$. Regarding this issue, our results showed that the main cause of starting KRT was fluid overload, with a significant 1.91 -fold increase risk (online suppl. Table 2) and a 13\% increased risk of mortality in those patients, which is similar to those found in other cohort studies $[21,29]$. In patients with established AKI, further fluid overloading has been linked with lower sur- vival and less renal recovery [30, 31]; limiting and resolving fluid overload might prompt earlier use of KRT. Our results reaffirm the importance of how the nephrologist's early intervention with fluid adjustment could positively change the trajectory of patients with AKI.

As we observed in our study, the use of diuretics was associated with a significant 1.23 -fold increase in the risk of starting KRT (Table 2). The use of diuretics in AKI has been controversial, and some benefits have been described; higher post-AKI furosemide doses had a protective effect against mortality [32]. In addition, starting diuretics at the cessation of CRRT contributed to the successful discontinuation of CRRT by reducing the volume overload risk in critically ill patients with AKI [33]. In contrast to a meta-analysis of 28 randomized trials, furosemide in patients with AKI or at risk for starting AKI did 
not have a lower mortality, reduced incidence of AKI, worsening of AKI, or decreased utilization of RRT [34]. We could explain the higher risk of KRT utilization in our study because patients with AKI were prescribed diuretics due to complications such as oliguria, anuria, or fluid overload.

Based on our results, none of the early nephrologist interventions reduced the risk of death in AKI patients. The multifactorial nature of AKI in hospitalized patients demands a multidisciplinary approach [35], where nephrologists can help in identifying early risk factors, adopting strategies to prevent the progression to more severe forms, and offering support renal therapy, not just KRT, when indicated. Ronco and Bellomo [36] in 1998 described the formal development of the specialty area called critical care nephrology, and the first aim was to recognize that the management of AKI in the ICU demands a multidisciplinary approach. Improving the quality of care provided to AKI patients, plausibly mitigating the cost of care, and improving short- and long-term outcomes are goals that have not been universally achieved. Therefore, understanding how the management of AKI may be amenable to quality improvement programs is needed.

In our study, we found that the use of vasopressors was associated with an increase of $25 \%$ in the probability of mortality. It is possible that this event is a reflection of the severity of the patients since it would represent a state of shock and hemodynamic instability that compromises perfusion of the tissues and is associated with a higher inhospital mortality [37] but may also be associated with decreased kidney recovery [38]. We also found that in AKI, the need of KRT increased the mortality risk, which may reflect a sicker group of patients. The AKI KDIGO 3 patients (with KRT) clearly demonstrated an increase in mortality, with adjusted ORs of 4.1 [39] compared with less severe grades (1 and 2). These data support the existence of the biological gradient between AKI severity and mortality. A short estimated 2.3-fold increase in the risk of death attributable to AKI has been reported [39]. The pathophysiology of AKI is sufficiently heterogeneous to account for morbidity and mortality on a wide range of timescales.

The strengths of our study are that we separate, for the first time, the effect of some nephrologist interventions on AKI, and with this, we were able to observe the importance of each intervention separately. To our knowledge, the results presented in this study have never been published. Several limitations should be noted. Because of the observational and retrospective nature of the study, no causal relationships could be established. The presented cohort may be associated with its own biases due to its design, and one of them is selection bias. We did not classify the etiology of AKI and did not measure another important nephrologist intervention, such as glucose control and type of sedation. The study was carried out in a single center, and the follow-up of 10 days was relatively short.

\section{Conclusion}

In conclusion, in AKI patients, early nephrology consultation, specifically fluid adjustment, could prevent the need for KRT and decrease the progression of AKI.

\section{Acknowledgements}

The authors would like to thank the medical, nursing, and allied health staff of the Nephrology Service of the Hospital Civil de Guadalajara for treating all patients with joy and kindness and the social service medicine interns, who are a source of inspiration.

\section{Statement of Ethics}

The study was approved by the Hospital Civil de Guadalajara Fray Antonio Alcalde Institutional Review Board (HCG 146/18) and was conducted in adherence with the Declaration of Helsinki. Informed and written consent was obtained from all the subjects.

\section{Conflict of Interest Statement}

The authors have no conflicts of interest to declare.

\section{Funding Sources}

No funding was used to make this protocol.

\section{Author Contributions}

J.S.C.I., P.M.A., and C.P.F. designed the protocol, collected the data, analyzed it, and wrote the manuscript. R.C.D.G., A.E.T.Q., F.R.A., G.N.B., R.M.G., J.G.R., F.Y.E., and G.G.G. analyzed it and wrote the manuscript. 


\section{References}

1 Bellomo R, Ronco C. Acute renal failure in the intensive care unit: adequacy of dialysis and the case for continuous therapies. Nephrol Dial Transplant. 1996;11(3):424-8.

2 Bellomo R, Ronco C, Kellum JA, Mehta RL, Palevsky P. Acute renal failure - definition, outcome measures, animal models, fluid therapy and information technology needs: the Second International Consensus Conference of the Acute Dialysis Quality Initiative (ADQI) Group. Crit Care. 2004;8:R204-12.

3 Bagshaw SM, Laupland KB, Doig CJ, Mortis G, Fick GH, Mucenski M, et al. Prognosis for long-term survival and renal recovery in critically ill patients with severe acute renal failure: a population-based study. Crit Care. 2005;9(6):R700-9.

4 Mehta RL, Pascaul MT, Soroko S, Savage DR, Himmelfarb J, Ikizler TA, et al. Spectrum of acute renal failure in the intensive care unit: the PICARD experience. Kidney Int. 2004;66: 1613-21.

5 Tolwani A. Continuous renal-replacement therapy for acute kidney injury. N Engl J Med. 2012;367(26):2505-14

6 Soares DM, Pessanha JF, Sharma A, Brocca A, Ronco C. Delayed nephrology consultation and high mortality on acute kidney injury: a meta-analysis. Blood Purif. 2017;43(1-3):5767.

7 Flores-Gama C, Merino M, Baranda F, Cruz DN, Ronco C, Vazquez-Rangel A. The impact of integrating nephrologists into the postoperative cardiac intensive care unit: a cohort study. Cardiorenal Med. 2013;3(1):79-88.

8 Jung Oh H, Lee MJ, Kim CH, Kim DY, Sun Lee H, Park JT, et al. The benefit of specialized team approaches in patients with acute kidney injury undergoing continuous renal replacement therapy: propensity score matched analysis. Crit Care. 2014;18(4):454.

9 Rizo-Topete LM, Rosner MH, Ronco C. Acute kidney injury risk assessment and the nephrology rapid response team. Blood Purif. 2017;43(1-3):82-8.

10 Joannidis M, Druml W, Forni LG, Groeneveld ABJ, Honore PM, Hoste E, et al. Prevention of acute kidney injury and protection of renal function in the intensive care unit: update 2017. Intensive Care Med. 2017;43(6):730-49.

11 Kellum JA, Lameire N, Aspelin P, Barsoum RS, Burdmann EA, Goldstein SL, et al. Kidney disease: Improving global outcomes (KDIGO) acute kidney injury work group. KDIGO clinical practice guideline for acute kidney injury. Kidney Int Supplements. 2012;2(1):1138.

12 Singer M, Deutschman CS, Seymour CW, Shankar-Hari M, Annane D, Bauer M, et al. The third international consensus definitions for sepsis and septic shock (Sepsis-3). JAMA. 2016 Feb 23;315(8):801-10.

13 Von Elm E, Altman DG, Egger M, Pocock SJ, Gøtzsche PC, Vandenbroucke JP, et al. The strengthening the reporting of observational studies in epidemiology (STROBE) statement: guidelines for reporting observational studies. Rev Esp Salud Publica. 2008;82(3): 251-9.

14 Fissell WH. Antimicrobial dosing in acute renal replacement. Adv Chronic Kidney Dis. 2013;20(1):85-93.

15 Cruz DN. Cardiorenal syndrome in critical care: the acute cardiorenal and renocardiac syndromes. Adv Chronic Kidney Dis. 2013; 20(1):56-66.

16 Matzke GR, Aronoff GR, Atkinson AJ Jr, Bennett WM, Decker BS, Eckardt KU, et al. Drug dosing consideration in patients with acute and chronic kidney disease-a clinical update from Kidney Disease: Improving Global Outcomes (KDIGO). Kidney Int. 2011;80(11): 1122-37.

17 Negi S, Koreeda D, Kobayashi S, Iwashita Y, Shigematu T. Renal replacement therapy for acute kidney injury. Ren Replace Ther. 2016; 2(1):31.

18 Eyler RF, Mueller BA, Medscape. Antibiotic dosing in critically ill patients with acute kidney injury. Nat Rev Nephrol. 2011;7(4):22635.

19 Negi S, Koreeda D, Kobayashi S, Iwashita Y, Shigematu T. Renal replacement therapy for acute kidney injury. Ren Replace Ther. 2016; 2(1):31.

20 Leaf DE, Waikar SS. IDEAL-ICU in context. Clin J Am Soc Nephrol. 2019;14(8):1264-7.

21 Salahuddin N, Sammani M, Hamdan A, Joseph M, Al-Nemary Y, Alquaiz R, et al. Fluid overload is an independent risk factor for acute kidney injury in critically Ill patients: results of a cohort study. BMC Nephrol. 2017; 18(1):45.

22 Ostermann M, Bellomo R, Burdmann EA, Doi K, Endre ZH, Goldstein SL, et al. Controversies in acute kidney injury: conclusions from a kidney disease: improving global outcomes (KDIGO) Conference. Kidney Int. 2020;98(2):294-309.

23 Hoste EA, Bagshaw SM, Bellomo R, Cely CM, Colman R, Cruz DN, et al. Epidemiology of acute kidney injury in critically ill patients: the multinational AKI-EPI study. Intensive Care Med. 2015;41(8):1411-23.

24 Ostermann M, Liu K, Kashani K. Fluid management in acute kidney injury. Chest. 2019; 156(3):594-603.

25 Prowle JR, Kirwan CJ, Bellomo R. Fluid management for the prevention and attenuation of acute kidney injury. Nat Rev Nephrol. 2014;10(1):37-47. Published online 12 November 2013.

26 Jentzer JC, Chawla LS. A clinical approach to the acute cardiorenal syndrome. Crit Care Clin. 2015;31(4):685-703.

27 Teixeira C, Garzotto F, Piccinni P, Brienza N, Iannuzzi M, Gramaticopolo S, et al. Fluid balance and urine volume are independent predictors of mortality in acute kidney injury. Crit Care. 2013;17(1):R14.
28 Wang N, Jiang L, Zhu B, Wen Y, Xi XM. Beijing Acute Kidney Injury Trial Workgroup. Fluid balance and mortality in critically ill patients with acute kidney injury: a multicenter prospective epidemiological study. Crit Care. 2015;19:371.

29 Vaara ST, Korhonen AM, Kaukonen KM, Nisula S, Inkinen O, Hoppu S, et al. Fluid overload is associated with an increased risk for 90-day mortality in critically ill patients with renal replacement therapy: data from the prospective FINNAKI study. Crit Care. 2012 Oct 17;16(5):R197.

30 Bellomo R, Bellomo R, Cass A, Cole L, Finfer S, Gallagher M, et al. An observational study fluid balance and patient outcomes in the randomized evaluation of normal vs. Augmented level of replacement therapy trial. Crit Care Med. 2012;40(6):1753-60.

31 Heung M, Wolfgram DF, Kommareddi M, $\mathrm{Hu}$ Y, Song PX, Ojo AO. Fluid overload at initiation of renal replacement therapy is associated with lack of renal recovery in patients with acute kidney injury. Nephrol Dial Transplant. 2012;27(3):956-61.

32 Grams ME, Estrella MM, Coresh J, Brower RG, Liu KD. National heart, lung, and blood institute acute respiratory distress syndrome network. Fluid balance, diuretic use, and mortality in acute kidney injury. Clin J Am Soc Nephrol. 2011;6(5):966-73.

33 Jeon J, Kim DH, Baeg SI, Lee EJ, Chung CR, Jeon $\mathrm{K}$, et al. Association between diuretics and successful discontinuation of continuous renal replacement therapy in critically ill patients with acute kidney injury. Crit Care. 2018;22(1):255.

34 Mehta RL, Pascual MT, Soroko S, Chertow GM, Group PS. Diuretics, mortality, and nonrecovery of renal function in acute renal failure. JAMA. 2002;288(20):2547-53.

35 Kashani K, Rosner MH, Haase M, Lewington AJP, O’Donoghue DJ, Wilson FP, et al. Quality improvement goals for acute kidney injury. Clin J Am Soc Nephrol. 2019;14(6):94153.

36 Ronco C, Bellomo R. Critical care nephrology: the time has come. Nephrol Dial Transplant. 1998;13(2):264-7.

37 Silversides JA, Pinto R, Kuint R, Wald R, Hladunewich MA, Lapinsky SE, et al. Fluid balance, intradialytic hypotension, and outcomes in critically ill patients undergoing renal replacement therapy: a cohort study. Crit Care. 2014;18(6):624.

38 Augustine JJ, Sandy D, Seifert TH, Paganini EP. A randomized controlled trial comparing intermittent with continuous dialysis in patients with ARF. Am J Kidney Dis. 2004;44(6): 1000-7.

39 Girling BJ, Channon SW, Haines RW, Prowle JR. Acute kidney injury and adverse outcomes of critical illness: correlation or causation? Clin Kidney J. 2020 Nov 18;13(2):13341. 